\title{
УЧЕТ АДСОРБЦИОННЫХ ЭФФЕКТОВ ПРИ ОПРЕДЕЛЕНИИ ИНДЕКСОВ УДЕРЖИВАНИЯ УГЛЕВОДОРОДОВ \\ В КАПИЛЛЯРНОЙ ГАЗОЖИДКОСТНОЙ ХРОМАТОГРАФИИ
}

\author{
(Представил О. Эйзен)
}

Основной причиной неудовлетворительной межлабораторной воспроизводимости индексов удерживания углеводородов на полярных неподвижных жидких фазах (НЖФ) является адсорбция на внутренней поверхности капиллярной колонки (KK) и на поверхности раздела НЖФ-газовая фаза $\left[{ }^{1-3}\right]$. Воспроизводимость индексов удерживания значительно улучшается при замене стандартов (н-алканов) на соединения, близкие по адсорбционным свойствам к исследуемым. Так, при анализе олефиновых углеводородов удобно использовать в качестве стандартов нормальные 1-алкены [2]. Однако выбор класса соединений в качестве стандартов в значительной степени произволен, что затрудняет широкое использование таких индексов удерживания для решения рутинных аналитических задач.

Цель настоящей работы - разработка методов расчета т. н. инвариантных индексов удерживания, межлабораторная воспроизводимость которых существенно лучше обычных $\left[{ }^{1,4}\right]$, с использованием в качестве определяющей величины эффективной толщины пленки НЖФ.

Чистый удерживаемый объем в равновесной хроматографии является суммой объемов удерживания на отдельных фазах хроматографического сорбента [5]:

$$
V_{N, i}=K_{l, i} V_{l}+K_{g l, i} S_{g l}+K_{l, i} K_{s, i} S_{a},
$$

где $S_{g l}$ и $S_{a}$ - поверхности раздела фаз газ-НЖФ и НЖФ-твердый носитель; $V_{l}$ - объем НЖФ в колонке; $K_{g l, i}$ и $K_{s, i}-$ константы распределения хроматографируемого вещества между поверхностью раздела газ-НЖФ и газовой фазы и между поверхностью раздела НЖФ-твердый носитель и НЖФ; $K_{l, i}-$ константа распределения хроматографируемого вещества между НЖФ и газовой фазой.

Разлагая относительную величину удерживания в ряд по $1 / V_{l}$, получаем [4]:

где

$$
\frac{V_{N, i}}{V_{N, j}}=V_{r, i j}=\frac{K_{l, i}}{K_{l, j}}\left(1+\frac{\lambda_{i, j}}{V_{l}}\right),
$$

$$
\lambda_{i, j}=\frac{S_{g l} K_{g l, i}+S_{a} K_{l, i} K_{s, i}}{K_{l, i}}-\frac{S_{g l} K_{g l, j}+S_{a} K_{l, j} K_{s, j}}{K_{l, j}} .
$$

Если предположить, что НЖФ распределяется сплошным слоем по всей внутренней поверхности .KК и что при изменении содержания НЖФ величина ее поверхности не изменяется, можно записать:

$$
S_{g l} \cong S_{a}, \quad V_{l}=S_{a} d_{f}
$$


где $d_{f}$ - эффективная толщина слоя НЖФ. Тогда (2) можно переписать в виде

$$
V_{r, i j}=\frac{K_{l, i}}{K_{l, j}}\left(1+\frac{\lambda_{i, j}^{\prime}}{d_{f}}\right)
$$

где

$$
\lambda_{i, j}^{\prime}=\frac{K_{g l, i}+K_{l, i} K_{s, i}}{K_{l, i}}-\frac{K_{g l, j}+K_{l, j} K_{s, j}}{K_{l, j}} .
$$

В отличие от $\lambda_{i, j}$ постоянная $\lambda_{i, j}^{\prime}$ является функцией хроматографических констант и не зависит от колоночных факторов.

Рассмотрим зависимость от $d_{f}$ индексов удерживания Ковача:

$$
I_{i}=100 n+100 \frac{\lg \left(V_{N, i} / V_{N, n}\right)}{\lg \left(V_{N, m} / V_{N, n}\right)},
$$

где $V_{N, n}$ - чистый объем удерживания $H$-алкана, молекула которого содержит $n$ углеродных атомов; $V_{N, m}$ - чистый объем удерживания н-алкана, молекула которого содержит $m=n+1$ углеродных атомов.

Разложив правую часть (5) в ряд по $1 / d_{f}$, получим

$$
I_{i}=100 n+100 \frac{\lg \left(K_{l, i} / K_{l, n}\right)}{\lg \left(K_{l, m} / K_{l, n}\right)}+\frac{\delta_{i}}{d_{f}},
$$

где

$$
\delta_{i}=\frac{\frac{K_{l, n}}{K_{l, i}} \lg \left(\frac{K_{l, m}}{K_{l, n}}\right) \lambda_{i n}^{\prime}-\frac{K_{l, n}}{K_{l, m}} \lg \left(\frac{K_{l, i}}{K_{l, n}}\right) \lambda_{m n}^{\prime}}{\ln 10\left(\lg \frac{K_{l, m}}{K_{l, n}}\right)^{2}}
$$

Выражение (6) близко к полученному в [4]. Перепишем уравнение (6) в виде

$$
I_{i}=I_{0, i}+\delta_{i} / d_{f},
$$

где $I_{0, i}-$ инвариантный индекс удерживания, равный

$$
I_{0, i}=100 n+100 \frac{\lg \left(K_{l, i} / K_{l, n}\right)}{\lg \left(K_{l, m} / K_{l, n}\right)} .
$$

$I_{0, i}$ является функцией констант распределения хроматографируемого вещества и стандартов между НЖФ и газовой фазой, поэтому эта величина не должна зависеть от адсорбции в хроматографической системе. Величина $\delta_{i}$ отражает вклад в индексы удерживания адсорбции хроматографируемого вещества и стандартов на межфазных границах.

В $\left[{ }^{2}\right]$ для определения инвариантных индексов удерживания использована зависимость

$$
I=I_{0, i}+a_{i} / k_{\mathrm{cT}},
$$

где $k_{\text {ст }}-$ коэффициент емкости стандартного соединения, $a_{i}-$ постоянная, характеризующая адсорбцию исследуемого вещества на границах фаз.

В качестве стандартного соединения выбирается вещество, адсорбция которого на межфазных границах пренебрежимо мала по сравнению с растворением в НЖФ, т. е. соединение, для которого справедливо уравнение

$$
V_{N, \mathrm{cт}}=K_{l, \mathrm{c \tau}} V_{l} \text {. }
$$


Однако следует иметь в виду, что коэффициент емкости зависит от соотношения объема НЖФ и свободного объема колонки $V_{M}$ :

$$
k_{\mathrm{cT}}=K_{l, \mathrm{cr}} \frac{V_{l}}{V_{M}} .
$$

Для КК можно записать

$$
V_{l}=\pi d_{c} L d_{f}, \quad V_{M}=\pi d_{c}^{2} L / 4,
$$

где $d_{c}-$ внутренний диаметр колонки, $L-$ длина колонки. Тогда (11) можно переписать в виде

$$
k_{\mathrm{cT}}=K_{l, \mathrm{c \tau}} \frac{4 d_{f}}{d_{c}},
$$

откуда следует, что зависимость (9) справедлива для КК с одинаковым внутренним диаметром. Зависимость (7) является более универсальной, так как она не требует применения стандартного соединения и справедлива для КК различного диаметра.

Возможность довольно точного определения толщины пленки прн нанесении НЖФ на капилляр статическим способом позволяет использовать зависимость (7) для определения $\delta_{i}$ и $I_{0, i}$.

\section{Әкспериментальная часть}

Стеклянные КК 1-8 изготовлены в Волгодонском филиале ВНИИПАВ. Капилляры вытягивались из стеклянных трубок на сконструированной в лаборатории установке. Марка стекла С-52-1. Длина колонок 50-60 м. НЖФ ПЭГ 20М наносили статическим способом под высоким давлением [6]. Эффективность колонок по тетрадекану составляла $1-2$ тысячи теоретических тарелок на метр. Необходимую концентрацию ПЭГ $20 \mathrm{M} \mathrm{в} \mathrm{растворителе} \mathrm{(метаноле)} \mathrm{для} \mathrm{получения} \mathrm{заданной} \mathrm{тол-}$ щины пленки определяли по формуле

$$
c_{f}=4 d_{f} /\left(d_{c}+6 d_{f}\right) .
$$

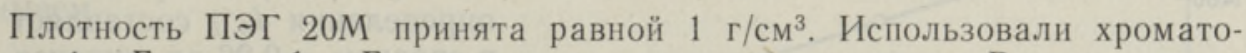
граф «Биохром-1». Газом-носителем служил водород. Времена удерживания исследуемых соединений измеряли электронным интегратором фирмы «Perkin Elmer M-1» (США) с точностью 1 с. Колонка 9 изготовлена в Институте химии АН ЭССР из стального капилляра длиной 100 м. НЖФ из 5,5\%-ного раствора ПЭГ $20 \mathrm{M}$ в хлороформе наносили динамическим способом. Газом-носителем служил гелий. Хроматограф - «Хром-4». Времена удерживания измеряли по расстояниям на диаграммной ленте. Деление газовых потоков составляло от 1:150 до 1:200. «Мертвое время» удерживания определяли расчетным способом по временам удерживания $н$-алканов.

\section{Результаты и обсуждение}

Из результатов экспериментального определения индексов удерживания некоторых изомеров тетрадеценов на НЖФ ПЭГ $20 \mathrm{M}$ при температуре термостата колонок $100^{\circ} \mathrm{C}$ (табл. 1) следует, что индексы удерживания, полученные на колонках 2 и 6 с толщиной пленки НЖФ 0,2 мкм, совпадают с ошибкой, не превышающей 0,9 ед., в то время как коэффициент емкости бензальдегида на этих колонках различается почти в три раза. Подобная ситуация наблюдается и в случае колонок 3 и 5 с $d_{f}=0,4$ мкм. 
Изменение индексов удерживания н-тетрадеценов в зависимости от толщины пленки НЖФ и диаметра колонок

\begin{tabular}{|c|c|c|c|c|c|c|c|c|c|c|c|c|c|}
\hline \multicolumn{4}{|c|}{$\begin{array}{l}\text { Показатели } \\
\text { для колонок }\end{array}$} & \multicolumn{10}{|c|}{ Изомеры н-тетрадецена } \\
\hline $\begin{array}{l}\text { 巳ิ } \\
\text { D̃ }\end{array}$ & 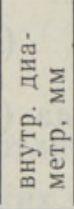 & 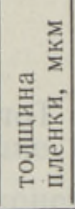 & 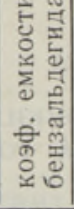 & 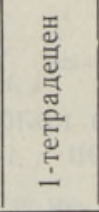 & 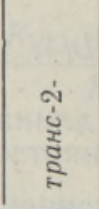 & 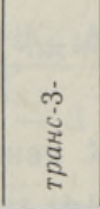 & 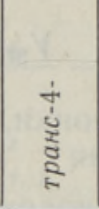 & 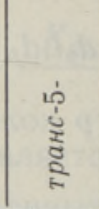 & 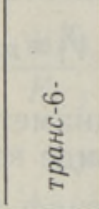 & 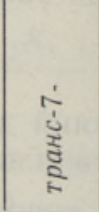 & 䓌 & 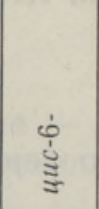 & 竎 \\
\hline $\begin{array}{l}1 \\
2 \\
3\end{array}$ & $\begin{array}{l}0,30 \\
0,30 \\
0,30 \\
0,30 \\
0,38 \\
0,49 \\
0,18 \\
0,30 \\
0,25\end{array}$ & $\begin{array}{l}0,11 \\
0,2 \\
0,4 \\
1,0 \\
0,4 \\
0,2 \\
- \\
-\end{array}$ & $\begin{array}{l}1,36 \\
3,54 \\
7,51 \\
21,0 \\
5,90 \\
1,36 \\
6,18 \\
6,32 \\
-\end{array}$ & $\begin{array}{l}1438,9 \\
1443,7 \\
1447,3 \\
1449,4 \\
1447,9 \\
1444,1 \\
1444,6 \\
1447,0 \\
1445,3\end{array}$ & $\begin{array}{l}1452,0 \\
1457,8 \\
1461,7 \\
1464,3 \\
1462,2 \\
1457,2 \\
1458,4 \\
1461,4 \\
1459,4\end{array}$ & $\begin{array}{l}1434,0 \\
1438,6 \\
1441,5 \\
1443,9 \\
1442,1 \\
1438,6 \\
1439,2 \\
1441,6 \\
1439,4\end{array}$ & $\begin{array}{l}1426,0 \\
1430,0 \\
1432,5 \\
1434,5 \\
: 432,9 \\
1429,4 \\
1430,4 \\
1432,4 \\
1438,8\end{array}$ & $\begin{array}{l}1424,9 \\
1428,5 \\
1431,2 \\
1432,8 \\
4431,8 \\
1428,2 \\
1429,2 \\
1430,9 \\
1428,9\end{array}$ & $\begin{array}{l}1422,1 \\
1425,8 \\
1428,3 \\
1430,2 \\
4428,8 \\
1496,5 \\
1426,2 \\
1428,1 \\
1426,8\end{array}$ & $\begin{array}{l}1421,0 \\
1424,8 \\
1427,0 \\
1428,7 \\
1427,6 \\
i 425,4 \\
1425,1 \\
1426,8 \\
1425,1\end{array}$ & $\begin{array}{l}1424,7 \\
1428,6 \\
1432,2 \\
1433,9 \\
1432,8 \\
1429,4 \\
: 429,8 \\
1431,6 \\
1450,2\end{array}$ & $\begin{array}{l}1421,7 \\
1425,5 \\
1428,5 \\
1430,5 \\
1429,0 \\
1426,1 \\
1426,1 \\
1428,2 \\
1426,2\end{array}$ & $\begin{array}{l}1419,4 \\
1423,1 \\
1426,4 \\
1428,0 \\
1427,1 \\
1424,0 \\
1424,0 \\
1425,9 \\
1424,5\end{array}$ \\
\hline
\end{tabular}

Из графика зависимости индексов удерживания от $1 / d_{f}$ для первых четырех колонок (рисунок) следует, что экспериментальные данные согласуются между собой с линейной зависимостью (7). Отрезки, отсекаемые прямыми на оси ординат, равны инвариантным индексам удерживания.

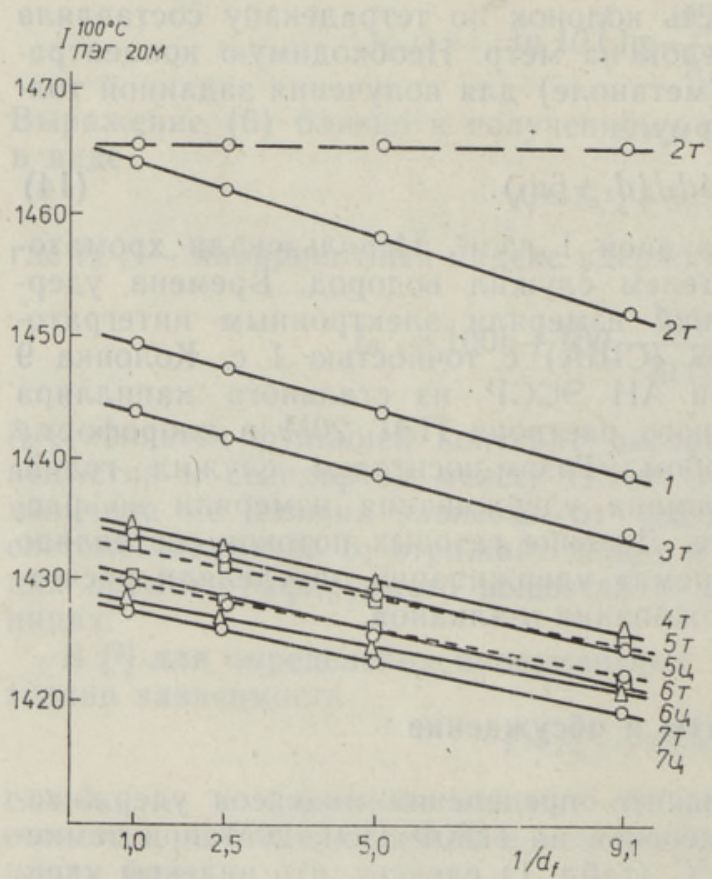

Зависимость индексов удерживания тетрадеценов от обратной величины толщины пленки НЖФПЭГ 20М. Цифры у линий - положенне двойной связи, ц. и т. - цис- и транс-изомеры, пунктир вверху - инвариантный индекс удерживания.
Нами были вычислены методом наименьших квадратов $I_{0, i}$ и $\delta_{i}$ некоторых тетрадеценов на ПЭГ 20M по индексам удерживания, полученным на колонках $1-4$ (табл. 2). Погрешность определения $I_{0, i}$ составляет в среднем $\pm 0,20$ ед. индекса удерживания, а $\delta_{i}-0,05$.

Таблица 2

Величины $\boldsymbol{I}_{0, i}$ и $\delta_{i}$ тетрадеценов, вычисленные по уравнению (7)

\begin{tabular}{l|c|c}
\hline $\begin{array}{c}\text { Изомеры } \\
\text { н-тетрадецена }\end{array}$ & $I_{0, i}$ & $\delta_{i}$ \\
\hline 1-Тетрадецен & 1450,5 & $-1,294$ \\
транс-2- & 1465,6 & $-1,509$ \\
транс-3- & 1444,8 & $-1,200$ \\
транс-4- & 1435,2 & $-1,022$ \\
транс-5- & 1433,6 & $-0,975$ \\
транс-6- & 1430,9 & $-0,986$ \\
транс-7- & 1429,5 & $-0,938$ \\
цис-5- & 1434,9 & $-1,148$ \\
цис-6- & 1431,3 & $-1,078$ \\
цис-7- & 1428,9 & $-1,072$ \\
& &
\end{tabular}


Величины $\hat{I}_{0, i}$, вычисленные для колонок 7-9

\begin{tabular}{|c|c|c|c|c|c|c|}
\hline \multirow{2}{*}{ Изомеры н-тетрадецена } & \multicolumn{2}{|c|}{ Колонка 7} & \multicolumn{2}{|c|}{ Колонка 8} & \multicolumn{2}{|c|}{ Колонка 9} \\
\hline & $I_{0, i}$ & $\Delta^{*}$ & $I_{0, i}$ & $\Delta$ & $I_{0, i}$ & $\Delta$ \\
\hline $\begin{array}{l}\text { 1-тетраде цен } \\
\text { транс-2-- } \\
\text { транс-3- } \\
\text { транс-4- } \\
\text { транс-5- } \\
\text { транс-6- } \\
\text { транс-7- } \\
\text { цис-5- } \\
\text { цис-6- } \\
\text { цис-7- }\end{array}$ & $\begin{array}{l}1450,5 \\
1465,3 \\
1444,7 \\
1435,0 \\
1433,6 \\
1430,7 \\
1429,4 \\
1435,0 \\
1431,0 \\
1428,9\end{array}$ & $\begin{array}{c}0 \\
0,3 \\
0,1 \\
0,2 \\
0 \\
0,2 \\
-0,1 \\
-0,1 \\
0,3 \\
0\end{array}$ & $\begin{array}{l}1450,5 \\
1465,5 \\
1444,8 \\
1435,2 \\
1433,5 \\
1430,8 \\
1429,3 \\
1434,7 \\
1,431,1 \\
1428,8\end{array}$ & $\begin{array}{l}0 \\
0,1 \\
0 \\
0 \\
0,1 \\
0,1 \\
0,2 \\
0,2 \\
0,2 \\
0,1\end{array}$ & $\begin{array}{l}1450,9 \\
1465,0 \\
1444,6 \\
1435,2 \\
1433,1 \\
1431,1 \\
1429,2 \\
1435,2 \\
1430,9 \\
1429,2\end{array}$ & $\begin{array}{r}-0,4 \\
-0,4 \\
0,2 \\
0 \\
0,5 \\
-0,2 \\
0,3 \\
-0,3 \\
0,4 \\
-0,3\end{array}$ \\
\hline
\end{tabular}

*. Разница между рассчитанными и приведенными в табл. 2 данными $I_{0, i}$.

Если адсорбция на поверхности раздела НЖФ-поверхность КК пренебрежимо мала по сравнению с адсорбцией на поверхности раздела газ-НЖФ, то величины $\delta_{i}$ будут зависеть только от типа НЖФ и анализируемого соединения. Поскольку полярная фаза ПЭГ 20M способствует экранировке активных центров поверхности стеклянного капилляра, можно считать, что найденные величины $\delta_{i}$ являются хроматографическими константами. Отрицательная величина $\delta_{i}$ свидетельствует о большей адсорбции н-алканов по сравнению с олефинами.

Продемонстрируем определение инвариантных индексов удерживания на КК 7 и 8 с неизвестной толщиной НЖФ, пользуясь индексами удерживания Ковача (табл. 1), определенными на этих колонках. Определим толщину пленки НЖФ по тетрадецену-1. Для колонки 7 имеем

$$
d_{f}=\frac{\delta_{i}}{I_{i}-I_{0, i}}=\frac{-1,294}{1444,6-1450,5}=0,22 \mathrm{M} \mathrm{KM}
$$

и для колонки $8-$

$$
d_{f}=\frac{-1,294}{1447,0-1450,5}=0,37 \text { мкм }
$$

Толщину пленки НЖФ можно вычислить более точно как среднее по всем изомерам. Среднее значение $d_{f}$ для колонки 9 получилось равным 0,23 мкм.

Инвариантные индексы удерживания, вычисленные исходя из полученных величин толщины пленки НЖФ, и их разница с индексами, вычисленными по данным четырех колонок (табл. 1, 3), показывают, что расхождения между значениями $I_{0, i}$ для стеклянных КК не превышают 0,3 ед., а для стальной $-0,5$ ед. Хорошая воспроизводимость инвариантных индексов удерживания на стеклянных и стальных КК свидетельствует о малом вкладе адсорбции на поверхности раздела НЖФ-поверхность капилляра в общее удерживание при использовании полярной НЖФ - ПЭГ 20 М. 


\section{Выводы}

1. Получено линейное уравнение, устанавливающее связь между индексами удерживания и обратной величиной эффективной толщины пленки неподвижной жидкой фазы в ГЖХ.

2. Инвариантные величины удерживания обладают хорошей межлабораторной и межоператорной воспроизводимостью. Они не зависят и от материала колонок (нержавеющая сталь, стекло).

\section{Л И Т Е РА Т У Р А}

1. Березкин В. Г. Влияние адсорбции анализируемых соединений на относительные величины удерживания в газожидкостной хроматографин. - Ж. анал. хим., 1985,40 , № 3, 555-563.

2. Soják, L., Berezkin, V. G., Janák, J. Effect of adsorption on the reproducibility of retention indices of hydrocarbons in capillary gas-liquid chromatography. J. Chromatogr., 1981, 209, 15-20.

3. Райкс И. А., Лейтен Н. А., Крамерс К. А., Березкин В. Г. Влияние адсорбционных явлений на воспроизводимость данных по удерживанию в капиллярных колонках. - Ж. анал. хим., 1974, 29, № 5, 858-862.

4. Березкин В. Г., Никитина Н. С., Фатеева В. М. Влияние адсорбцни на межфазных границах неподвижной жидкой фазы на относительные величины удерживания в распределительной хроматографин. - Докл. АН СССР, 1973, 229, № $5,1131-1134$.

5. Березкин В. Г., Похомов В. П., Татаринский В. С., Фатеева В. М. Изучение вклада адсорбции на межфазных границах в удерживаемый объем в газожидкостнотвердофазной хроматографии. - Докл. АН СССР, 1968, 180, № 5, 1135-1138.

6. Ilkova, E. L., Mistryukov, E. A. A simple versatile method for coating of glass capillary columns. - J. Chromatogr. Sci., 1971, 9, 569-570.

Волгодонский филиал ВНИИПАВ

Поступила в редакцию $17 /$ VI 1985

\section{Ннститут нефтехимического синтеза} Академии наук СССР

Институт химии

Академии наук Эстонской ССР

G. GUSSEV, Silvia RANG, V. BERJOZKIN, Anne ORAV

\section{ADSORPTSIOONIEFEKTID SUSIVESINIKE RETENTSIOONI- INDEKSITE MÄÄRAMISEL GAASI-VEDELIKKAPILLAAR- KROMATOGRAAFIAS}

On tuletatud lineaarne võrrand väljendamaks seost logaritmiliste retentsiooniindeksite ja liikumatu vedelfaasi (polüetüleenglükool 20M) efektiivse kihi paksuse pöördväärtuse vahel. On näidatud, et nn. invariantsete retentsiooniindeksite kasutamine retentsiooni parameetritena parandab tunduvalt erinevate operaatorite ja laboratooriumide mõōtmistulemuste reprodutseeritavust. Invariantsed retentsiooniindeksid ei sōltu kolonni materjalist (klaas, roostevaba teras).

\section{G. GUSSEV, Silvia RANG, V. BERYOZKIN, Anne ORAV}

\section{THE ADSORPTION EFFECT IN THE DETERMINATION OF RETENTION INDICES OF HYDROCARBONS IN CAPILLARY GAS-LIQUID CHROMATOGRAPHY}

The linear equation between the retention indices and the reciprocal value of the effective film thickness of the stationary liquid phase (polyethylene glycol 20M) in capillary gas-liquid chromatography has been derived. The use of invariant retention indices decreases the unreproducibility in the determination of the retention indices measured by different operators and in different laboratories. The invariant retention indices do not depend on the column material (glass, stainless steel). 